\title{
Approximating fractional derivatives in the perspective of system control
}

\author{
J.A. Tenreiro Machado • Alexandra Galhano
}

\begin{abstract}
The theory of fractional calculus goes back to the beginning of the theory of differential calculus, but its application received attention only recently. In the area of automatic control some work was devel- oped, but the proposed algorithms are still in a re- search stage. This paper discusses a novel method, with two degrees of freedom, for the design of frac- tional discrete-time derivatives. The performance of several approximations of fractional derivatives is in- vestigated in the perspective of nonlinear system con- trol.
\end{abstract}

Keywords Fractional calculus, Control,Discretization schemes

derivatives (FDs) in the discrete-time domain. The performance of the resulting algorithms is analyzed

\section{Introduction}

Fractional calculus (FC) is a natural extension of the classical mathematics. The fundamental aspects of the fractional calculus theory and the study of its properties can be addressed in references [1-3]. In what concerns the application of FC concepts, we can mention a large volume of research about viscoelasticity and damping, biology, signal processing, diffusion and wave propagation, modeling, identification and control [4-13].

Several researchers on automatic control proposed algorithms based on the frequency [7, 9] and the discrete-time [11-13] domains. This article introduces a novel method to implement fractional when adopted in the control of nonlinear systems. In this line of thought, the paper is organized as follows. Sections 2 and 3 develop the novel method of FD discrete-time approximation and investigate its performance in the control of a nonlinear system, respectively. Finally, Sect. 3 draws the main conclusions.

\section{On the generalization of fractional discrete-time control algorithms}

The Grünwald-Letnikov definition of a FD of order $\alpha$ of the signal $x(t), D^{\alpha} x(t)$, is given by

$$
D^{\alpha}[x(t)]=\lim _{h \rightarrow 0} \frac{1}{h^{\alpha}} \sum_{k=0}^{\infty}(-1)^{k} \frac{\Gamma(\alpha+1) x(t-k h)}{\Gamma(k+1) \Gamma(\alpha-k+1)}
$$


where $r$ is the gamma function and $h$ is the time increment. This formulation inspired the discrete-time FD calculation, by approximating the time increment $h$ through the sampling period $T$, yielding the equation in the $z$ domain:

$$
\begin{aligned}
Z\left\{D^{\alpha}[x(t)]\right\} & \approx \frac{1}{T^{\alpha}} \sum_{k=0}^{\infty} \frac{(-1)^{k} \Gamma(\alpha+1)}{k ! \Gamma(\alpha-k+1)} z^{-k} X(z) \\
& =\left(\frac{1-z^{-1}}{T}\right)^{\alpha} X(z)
\end{aligned}
$$

where $X(z)=Z\{x(t)\}$. In fact, expression (2) represents the Euler (or first backward difference) approximation in the so-called $s \rightarrow z$ conversion schemes. Other possibilities often adopted in control system design consist in the Tustin (or bilinear) and Simpson rules. The generalization to non-integer exponents of these conversion methods lead to the non-rational $z$-formulae:

$$
\begin{aligned}
& s^{\alpha} \approx\left[\frac{1}{T}\left(1-z^{-1}\right)\right]^{\alpha}=\left[H_{0}\left(z^{-1}\right)\right]^{\alpha} \\
& s^{\alpha} \approx\left(\frac{2}{T} \frac{1-z^{-1}}{1+z^{-1}}\right)^{\alpha}=\left[H_{1}\left(z^{-1}\right)\right]^{\alpha}
\end{aligned}
$$$$
\text { (3a) } \quad 9
$$

where $H_{0}\left(z^{-1}\right)$ and $H_{1}\left(z^{-1}\right)$ are often called generating approximants of zero and first order (i.e., the Euler and Tustin rules), respectively.

In order to get rational expressions the approximants need to be expanded into Taylor series and the final algorithm corresponds to a truncated series or to a rational Pade fraction.

We can obtain a family of fractional differentiators by the generating functions $H_{0}\left(z^{-1}\right)$ and $H_{1}\left(z^{-1}\right)$ weighted by the factors $p$ and $1-p$, yielding:

$$
H_{a v}\left(z^{-1} ; p\right)=p H_{0}\left(z^{-1}\right)+(1-p) H_{1}\left(z^{-1}\right)
$$

For example, the Al-Alaoui operator corresponds to an interpolation of the Euler and Tustin integration

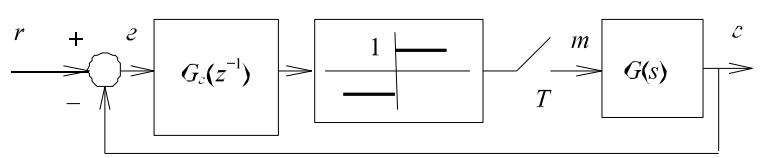

Fig. 1 The $D^{1 / 2}$ controller for a system with a nonlinearity in the forward loop

where $(q, p)$ are two tuning degrees of freedom, corresponding $q$ to the order of the averaging expression and $p$ to the weighting factor. For example, when $q=\{-1,0,1\}$, we get the well-known expressions for the $\{$ harmonic, geometric, arithmetic $\}$ averages, respectively.

Bearing these ideas in mind [23], we decided to examine the expression resulting from (5), for distinct values of $(q, p)$.

Tables 1 and 2 depict the coefficients of a second-

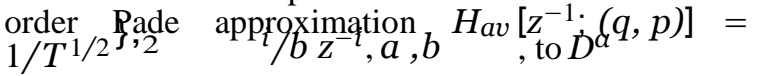

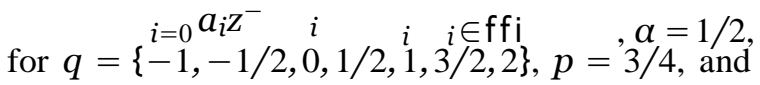
$q=\{-1,0,1,2\}, p=1 / 2$, respectively.

In order to test the performance of the expressions the usual method is to examine either the frequency domain, by comparing the Bode plots, or the time domain, by comparing the step response. Nevertheless, often the differences are negligible and, furthermore, do not have a direct translation to control system performance. Therefore, in our study we decided to test the approximations by analyzing the time response $c(t)$ for a reference unit input step $r(t)$ stimHhation of the closed loop control system represented 作 rules with weighting factor $p=3 / 4[14,15]$. These approximation methods have been studied by several researchers [16-23] and motivated a novel averaging method [24] based on the generalized formula of averages (often called average of order $q \in \mathrm{ffi}$ ):

$$
\begin{aligned}
H_{a v}\left[z^{-1} ;(q, p)\right]= & \left\{p\left[H_{0}\left(z^{-1}\right)\right]^{q}\right. \\
& \left.+(1-p)\left[H_{1}\left(z^{-1}\right)\right]^{q}\right\}^{\frac{1}{q}}
\end{aligned}
$$


ments the controller for several values of $(q, p)$, with $\alpha=1 / 2$, and a system with transfer function $G(s)=1 /[s(s+1)]$. The inclusion of the on-off nonlinearity (i.e., the unit relay) in the forward loop leads to the simplification of the analysis because the controller gain is not relevant and is not necessary to tune, but, on the other hand, we have a stringent dynamic test that stimulates both the transient and the steady-state behavior.

Figure 2 depicts the closed-loop step response $c(t)$ for $q=\{-1,-1 / 2,0,1 / 2,1,3 / 2,2\}$ and $p=3 / 4$.

Figure 3 presents the step response $c(t)$ for $q=$ $\{-1,0,1,2\}$ and $p=1 / 2$ In both cases, we con- 
Table 1 Coefficients of the $D^{\alpha}, \alpha=1 / 2$, Pade fraction approximation for $p=3 / 4$

\begin{tabular}{lllllll}
\hline$q$ & $a_{0}$ & $a_{1}$ & $a_{2}$ & $b_{0}$ & $b_{1}$ & $b_{2}$ \\
\hline-1 & 0.76597491 & 6.026479067 & -7.984934523 & 1 & 1.054474431 & -7.609965244 \\
$-1 / 2$ & 1.324947376 & 3.621337338 & -6.248676637 & 1 & -0.000172433 & -5.761497326 \\
0 & 1.465218175 & 2.188544252 & -4.788397277 & 1 & -0.804204046 & -4.788397277 \\
$1 / 2$ & 1.734358402 & 1.669224885 & -4.589703269 & 1 & -1.164316112 & -4.184478992 \\
1 & 1.787304546 & 1.2339426 & -4.155138823 & 1 & -1.36761081 & -3.765228028 \\
$3 / 2$ & 1.779700183 & 0.975910656 & -3.828827863 & 1 & -1.464217163 & -3.447457027 \\
2 & 1.723916925 & 0.840452121 & -3.56477439 & 1 & -1.480178572 & -3.188431144 \\
\hline
\end{tabular}

Table 2 Coefficients of the $D^{\alpha}, \alpha=1 / 2$, Pade fraction approximation for $p=1 / 2$

\begin{tabular}{rllllll}
\hline \multicolumn{1}{c}{$a_{0}$} & $a_{1}$ & $a_{2}$ & $b_{0}$ & $b_{1}$ & $b_{2}$ \\
\hline-1 & 0.218192312 & 2.932612925 & -3.758174112 & 1 & 1.815189846 & -8.845101094 \\
0 & 1.645896792 & 2.146868296 & -5.087608366 & 1 & -1.403319874 & -4.278151638 \\
1 & 1.667798875 & 1.603885503 & -4.470002812 & 1 & -1.607438339 & -3.703075415 \\
2 & 1.488976016 & 1.498893033 & -4.035502783 & 1 & -1.52197687 & -3.29498733 \\
\hline
\end{tabular}

$\{0.1,0.2,0.3\}$, in order to test also the robustness for fast versus slow sampling controllers. We verify that:

- In general, the order $q=1$ is the one that produces the best results.

- The sampling period $T=0.1$ leads to a good performance, while the results degrade considerably for larger values of $T$.

- The difference between $p=3 / 4$ and $p=1 / 2$ seems to be negligible, particularly in the case of $q=1$.

The characterization of a 'good' response can be accomplished in terms of optimization terms. In the present case such strategy was not necessary due to the simplicity of the test. Therefore, a 'good' response is simply the one that has a fast transient and a low amplitude steady-state limit cycle.

An interesting experiment is to analyze the tuning effect of $(q, p)$ in a system with delay $d$. Therefore, we evaluate the system step response in the case of the transfer function $G(s)=e^{-s d} /[s(s+1)]$, $d=\{0,0.5\}$, for $q=\{-1,0,1,2\}$ and $p=\{1 / 2,3 / 4\}$. Figure 4 presents the step time responses $c(t)$ for $q=\{-1,0,1,2\}, p=1 / 2$, when $T=0.1$. We have almost similar responses for $p=3 / 4$ and, therefore, the corresponding charts are not presented.
We observe that now the best case occurs when $q=-1$, leading to a peak time $t_{p}=3.02 \mathrm{~s}$ and an percent overshoot os $\%=28 \%$, while the rest of the cases have worst characteristics, namely $t_{p}=3.12 \mathrm{~s}$ and os $\%=38 \%$.

The unit relay, in the forward path of the closed loop system, avoids the requirement for the controller gain tuning, but, on the other hand, it may obscure slightly the comparison of the transient. Therefore, another direction of research is to consider the case of having a linear system. In this perspective, twe evaluate the system step response in the case a $D$ controller, implemented through $G_{c}\left(z^{-1}\right)=K H_{a v}\left[z^{-1} ;(q, p)\right]$, where $K$ represents the gain, and $q=\{-1,0,1,2\}$ and $p=1 / 2$, without considering any nonlinearity in the forward path, and of the transfer function $G(s)=$ $e^{-s d} /[s(s+1)], d=0.5$.

Figure 5 presents the step time responses $c(t)$ and the control effort $m(t)$ for $q=\{-1,0,1,2\}, p=1 / 2$, $T=0.1$, when $K=\{1,2,5\}$. For example, if we consider 'good' to have a slight overshoot in the output response $c(t)$, we get the cases $q=-1, K=5$ and $q=\{0,1,2\}, K=2$ which seem similar. However, from the control effort $m(t)$, the case $q=-1, K=5$ leads to a smother signal, while the other three cases impose an oscillatory command that results in the actuator stress. While the adoption of such a simple con- 
Fig. 2 Closed-loop step response $c(t)$ for a $D^{1 / 2}$ controller with

$q=\{-1,-1 / 2,0,1 / 2,1$, $3 / 2,2\}$ and $p=3 / 4$,

$G(s)=1 /[s(s+1)]$, and the unit relay in the forward loop
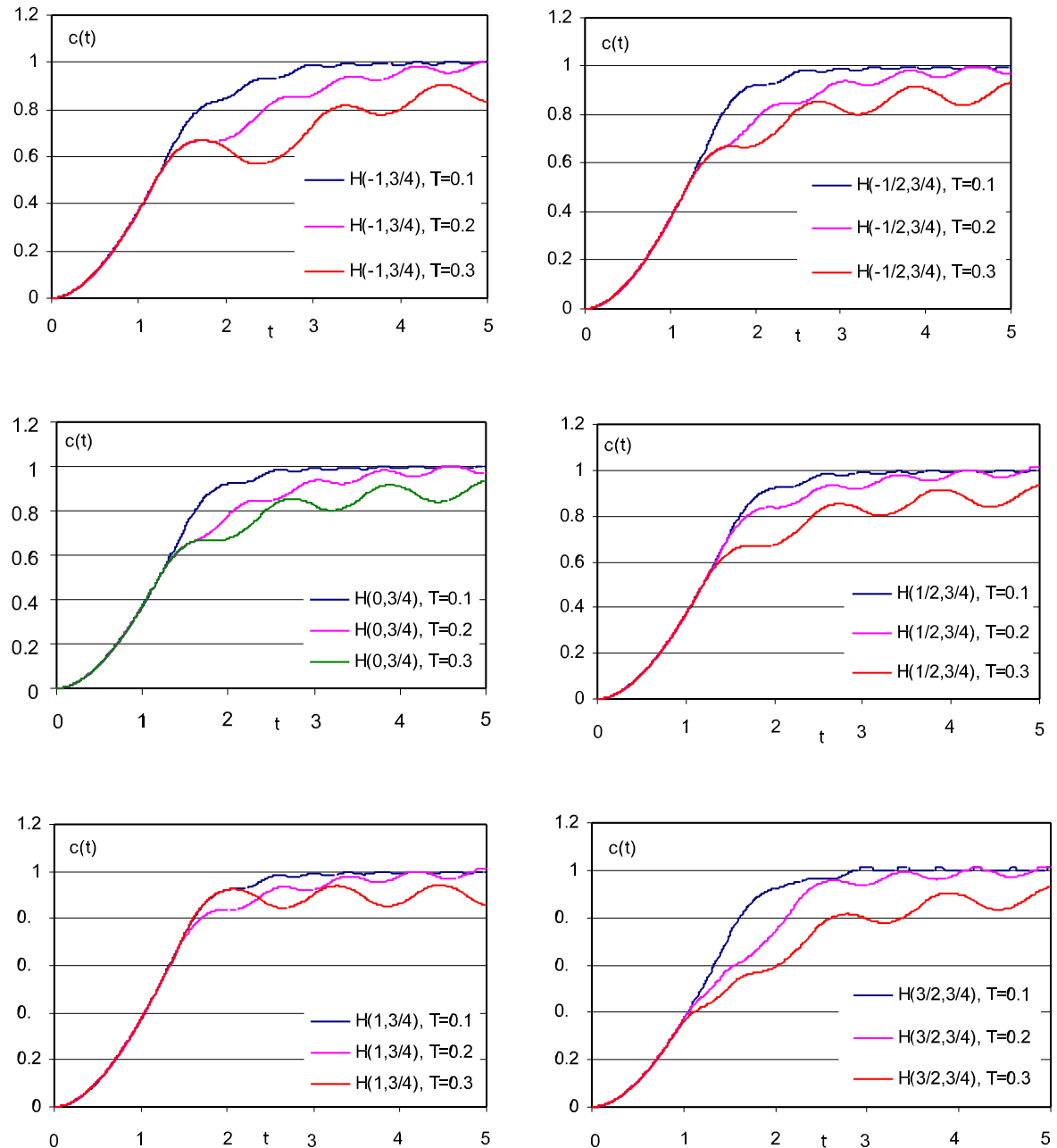

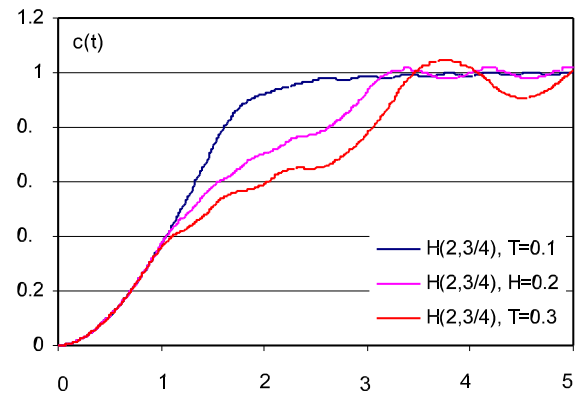

troller and the numerical values $p=1 / 2$ and $\alpha=1 / 2$ is discussible, we verify that the parameter $q$ gives an extra degree of freedom for the control design.

In conclusion, in this article we extended the optimization control problem. In fact, with the classical PID (Proportional, Integral, Differential) algorithm, we have three parameters to adjust, namely the proportional, integral and differential gains $\left\{K_{p}, K_{i}, K_{d}\right\}$. For the fractional PID algorithm [11-13], so-called $P I^{\beta} D^{\alpha}, \beta, \alpha>0$, we have five parameters $\left\{K_{p}, K_{i}\right.$, $\left.\beta, K_{d}, \alpha\right\}$. In this study, we verify that it is advisable to consider also the discretization scheme and the 
Fig. 3 Closed-loop step response $c(t)$ for a $D^{1 / 2}$ controller with $q=\{-1,0,1,2\}$ and $p=1 / 2, G(s)=$ $1 /[s(s+1)]$, and a unit relay in the forward loop

Fig. 4 Closed-loop step response $c(t)$ for a $D^{1 / 2}$ controller with $q=\{-1,0,1,2\}$ and $p=1 / 2, T=0.1$,

$G(s)=e^{-s d} /[s(s+1)]$, $d=\{0,0.5\}$, and the unit relay in the forward loop
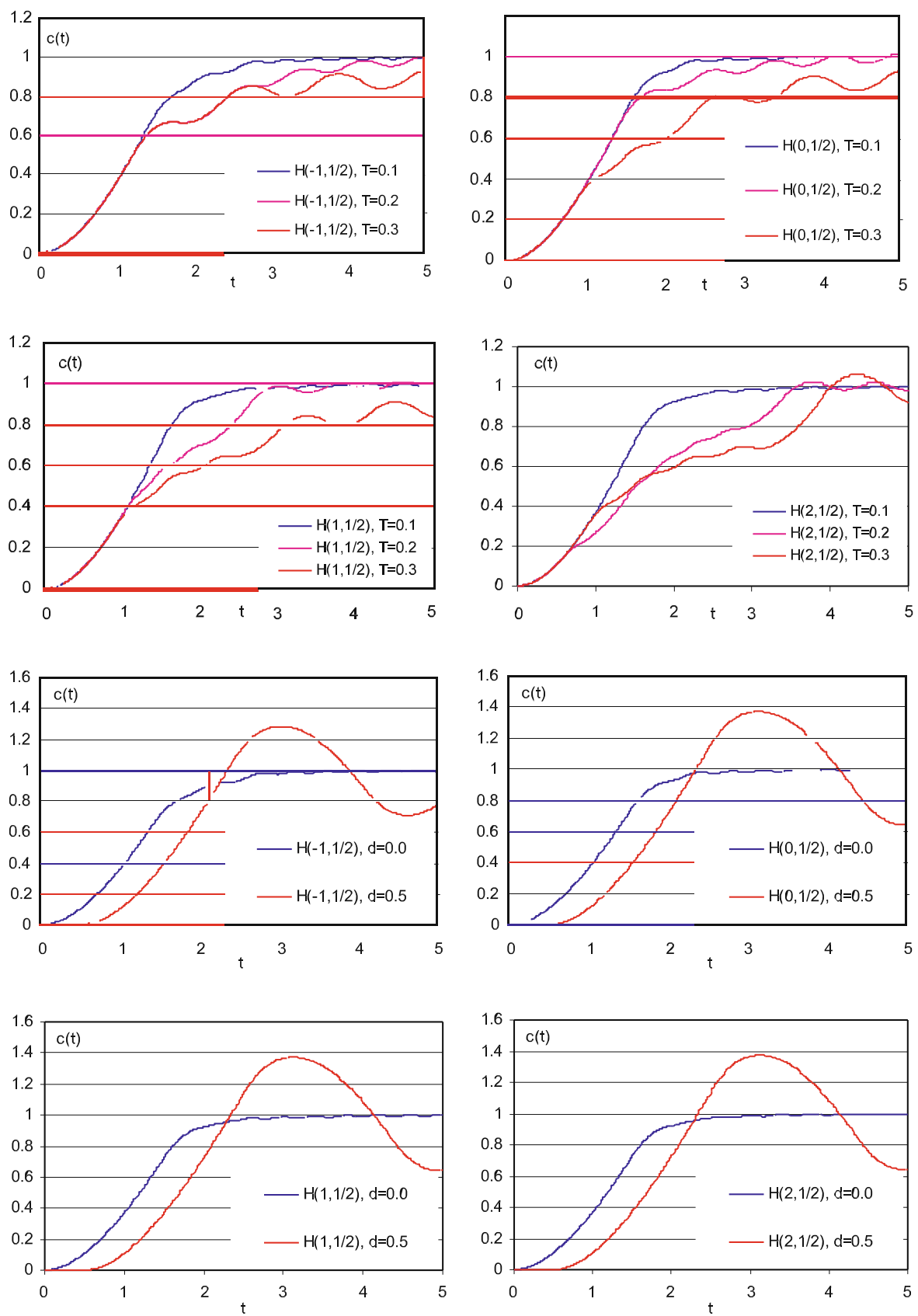

approximation formula leading to seven tuning parameters $\left\{K_{p}, K_{i}, \beta, K_{d}, \alpha, p, q\right\}$. The development of automatic optimal tuning strategies, capable of tacking advantage of all degrees of freedom, needs further study. Also, the adoption of different optimization indices, for distinct classes of dynamical systems, and the relationship with the parameters will be a matter of future research.

\section{Conclusions}

In this paper a novel method for the discrete-time FD approximation was presented and evaluated. The new algorithm adopts the time domain and generates a family of possible approximations, having two distinct degrees of freedom, namely, the order of the averaging and the weight of the generating functions. The prop- 
Fig. 5 Closed-loop step response $c(t)$ and control effort $m(t)$ for a $D^{1 / 2}$ controller with $q=\{-1,0,1,2\}, p=1 / 2$, $T=0.1$, controller gains $K=\{1,2,5\}$,

$G(s)=e^{-s d} /[s(s+1)]$, $d=0.5$, without any nonlinearity in the forward loop
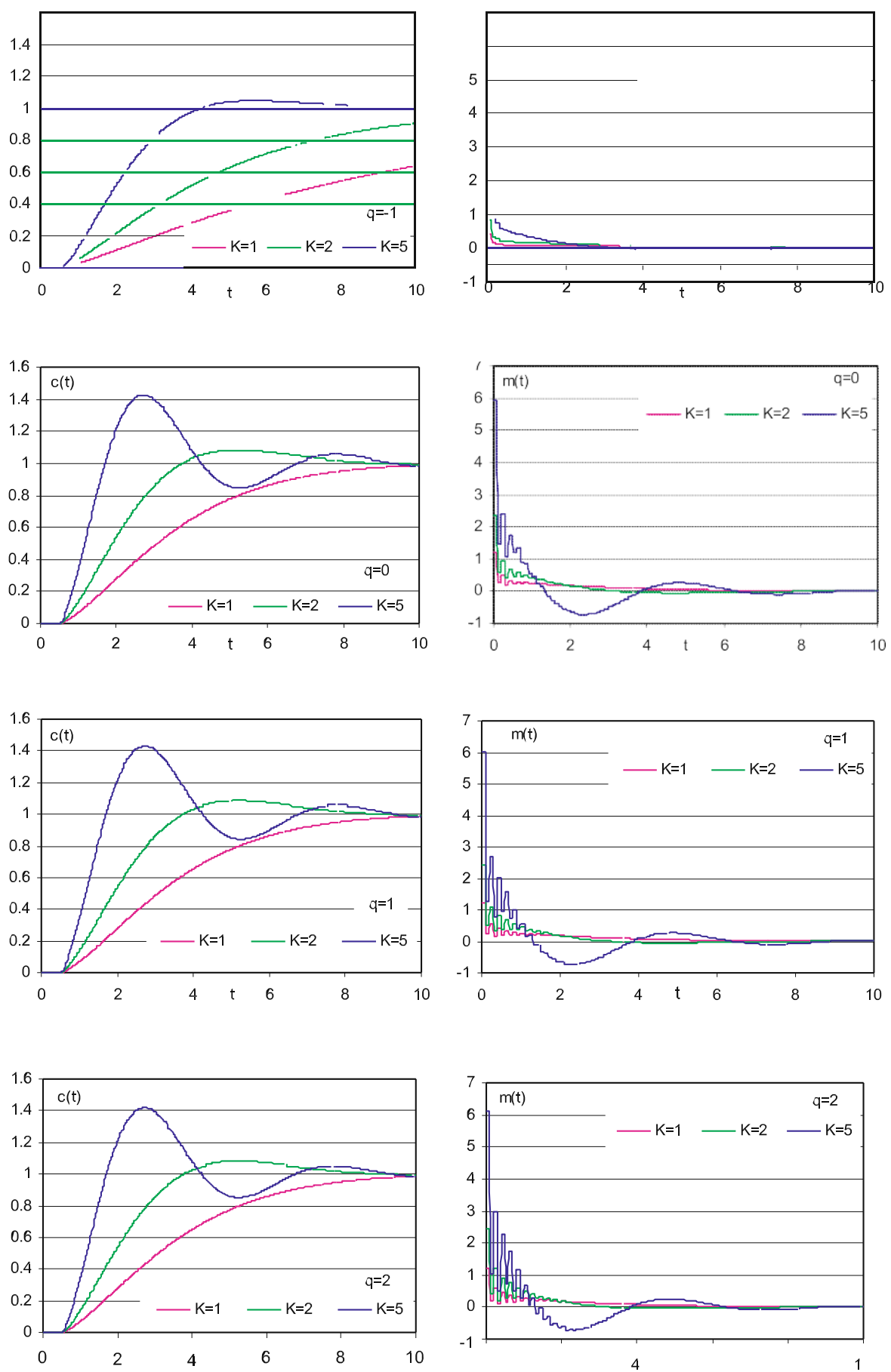

erties of several expressions were studied for a simple nonlinear system. The time response of the closedloop system was analyzed and the robustness for different sampling periods was tested. The conclusions are consistent and motivate an extensive test of all possibilities opened by the extra degrees of freedom.

\section{References}

1. Oldham, K.B., Spanier, J.: The Fractional Calculus: Theory and Application of Differentiation and Integration to Arbitrary Order. Academic Press, San Diego (1974) 
2. Samko, S.G., Kilbas, A.A., Marichev, O.I.: Fractional Integrals and Derivatives: Theory and Applications. Gordon and Breach, New York (1993)

3. Miller, K.S., Ross, B.: An Introduction to the Fractional Calculus and Fractional Differential Equations. Wiley, New York (1993)

4. Bagley, R.L., Torvik, P.J.: Fractional calculus-a different approach to the analysis of viscoelastically damped structures. AIAA J. 21(5), 741-748 (1983)

5. Nigmatullin, R.R.: The realization of the generalized transfer equation in a medium with fractal geometry. Phys. Status Solidi 133, 425-430 (1986)

6. Le Méhauté, A.: Fractal Geometries: Theory and Applications. Penton, Cleveland (1991)

7. Oustaloup, A.: La commande CRONE: commande robuste d'ordre non entier. Hermes, Paris (1991)

8. Anastasio, T.J.: The fractional-order dynamics of brainstem vestibulo-oculomotor neurons. Biol. Cybern. 72(1), 69-79 (1994)

9. Oustaloup, A.: La dérivation non entier: théorie, synthèse et applications. Hermes, Paris (1995)

10. Mainardi, F.: Fractional relaxation-oscillation and fractional diffusion-wave phenomena. Chaos Solitons Fractals 7(9), 1461-1477 (1996)

11. Machado, J.T.: Analysis and design of fractional-order digital control systems. Syst. Anal. Model. Simul. 27(2-3), 107-122 (1997)

12. Podlubny, I.: Fractional-order systems and $P I^{\lambda} D^{\mu}$ controllers. IEEE Trans. Automat. Contr. 44(1), 208-213 (1999)

13. Podlubny, I.: Fractional Differential Equations. Academic Press, San Diego (1999)

14. Al-Alaoui, M.A.: Novel digital integrator and differentiator. Electron. Lett. 29(4), 376-378 (1993)
15. Al-Alaoui, M.A.: Filling the gap between the bilinear and the backward-difference transforms: an interactive design approach. Int. J. Electr. Eng. Educ. 34(4), 331-337 (1997)

16. Smith, J.M.: Mathematical Modeling and Digital Simulation for Engineers and Scientists, 2nd edn. Wiley, New York (1987)

17. Chen, Y.Q., Moore, K.L.: Discretization schemes for fractional-order differentiators and integrators. IEEE Trans. Circuits Syst.-I. Fundam. Theory Appl. 49(3), 363-367 (2002)

18. Tseng, C.C.: Design of fractional order digital fir differentiators. IEEE Signal Process. Lett. 8(3), 77-79(2001)

19. Vinagre, B.M., Chen, Y.Q., Petras, I.: Two direct Tustin discretization methods for fractional-order differentiator/integrator. J. Franklin Inst. 340(5), 349-362 (2003)

20. Chen, Y.Q., Vinagre, B.M.: A new IIR-type digital fractional-order differentiator. Signal Process. 83(11), 2359-2365 (2003)

21. Chen, Y.Q., Vinagre, B.M., Podlubny, I.: Continued fraction expansion approaches to discretizing fractional order derivatives - an expository review. Nonlinear Dyn. 38(14), 155-170 (2004)

22. Barbosa, R.S., Machado, J.T., Ferreira, I.M.: Least-squares design of digital fractional-order operators. In: Proceedings of the First IFAC Workshop on Fractional Differentiation and Its Applications, Bordeaux, France, pp. 434-439 (2004)

23. Barbosa, R.S., Machado, J.T., Silva, M.F.: Time domain design of fractional differintegrators using least squares approximations. Signal Process. 86(10), 2567-2581 (2006)

24. Machado, J.T., Galhano, A.: A new method for approximating fractional derivatives: application in non-linear control. In: ENOC 2008 - 6th EUROMECH Conference, Saint Petersburg, Russia (2008) 